Classification

Physics Abstracts

$07.10-81.70-62.50$

\title{
Description du canon à gaz DEMETER et des chaînes de mesures associées
}

\author{
P. Chartagnac et B. Jimenez \\ Centre d'Etudes de Gramat, 46500 Gramat, France \\ (Reçu le 30 novembre 1983, révisé le 11 avril 1984, accepté le 16 mai 1984)
}

\begin{abstract}
Résumé. - L'ensemble expérimental qui est décrit dans cet article est destiné à l'étude du comportement des solides en régime de choc plan. Il est constitué du canon à gaz comprimé DEMETER et de plusieurs chaînes de mesures. Le canon utilisant de l'air ou de l'hélium comme gaz moteur, peut propulser des " projectiles » de $110 \mathrm{~mm}$ de diamètre avec une vitesse continûment programmable de $100 \mathrm{~m} / \mathrm{s}$ à $1150 \mathrm{~m} / \mathrm{s}$ et une reproductibilité de $1 \%$. Les chaînes de mesures implantées sur le canon et reliées à un calculateur permettent d'obtenir des signaux de contrainte in situ ou de vitesse matérielle avec une résolution temporelle de quelques dizaines de nanosecondes lorsque l'on utilise des jauges et de quelques nanosecondes dans le cas des mesures interférométriques.
\end{abstract}

\begin{abstract}
The experimental set-up described in this paper is devoted to solid state shock wave physics. It includes a gas gun (DEMETER) and several data acquisition channels. The gun is driven by compressed air or helium and launches $110 \mathrm{~mm}$-diameter projectiles with continuously programmable velocities ranging from $100 \mathrm{~m} / \mathrm{s}$ to $1150 \mathrm{~m} / \mathrm{s}$ and a reproducibility of $1 \%$. The associated data acquisition channels are connected with a computer and deliver in situ stress or particle velocity profiles with an accuracy of a few tens of nanoseconds when using gauges and a few nanoseconds when using an interferometric technique.
\end{abstract}

\section{Introduction}

Les recherches sur la compression par choc des solides ont commencé vers 1940 , fortement stimulées par des applications militaires. Cependant, l'onde de choc est aussi un outil d'investigation de portée générale puisqu'elle permet des états extrêmes de pression et de température. C'est dans le domaine de la physique de l'état solide que les ondes de choc ont été le plus largement employées, mais on peut citer aussi des applications fructueuses en géophysique et en métallurgie. Parmi toutes les géométries d'ondes envisageables, le choc plan présente un intérêt particulier car il réduit le nombre des variables physiques. Les premières expérimentations en choc plan impliquaient des édifices pyrotechniques, mais la précision requise aujourd'hui incite à abandonner les explosifs au profit de la solution «lanceur». Dans la famille des "lanceurs", le canon à gaz comprimé tient une place privilégiée liée à l'étendue de ses possibilités, à sa grande reproductibilité et à sa rapidité de mise en ouvre.

Cette publication décrit le lanceur à gaz DEMETER installé dans le laboratoire de dynamique des matériaux du CEG, ainsi que les chaînes de mesures asso- ciées. Après avoir rappelé les grands principes de conception dans le paragraphe 2 , nous décrivons le canon dans le paragraphe 3 et donnons ses performances dans le paragraphe 4 . Les chaînes de mesures sont présentées succinctement en dernier lieu.

\section{Principes de conception du canon à gaz.}

Comme le montre la figure 1, un canon à gaz est essentiellement constitué d'un réservoir de gaz moteur à ouverture ultra-rapide, d'un tube de lancement, d'une chambre porte-cible et d'un dispositif de récupération des éclats. Le projectile est constitué d'un sabot poussant un disque baptisé « impacteur». A l'issue de sa course dans le tube de lancement, le projectile frappe l'échantillon instrumenté (cible) fixé dans la chambre porte-cible. Pour induire un choc plan, la face avant de l'échantillon et de " l'impacteur » doivent être parallèles.

Dans un échantillon donné, l'intensité du choc et sa durée ne dépendent que de la pression motrice, du poids du projectile, de la polaire de choc et de l'épaisseur de «l'impacteur». 

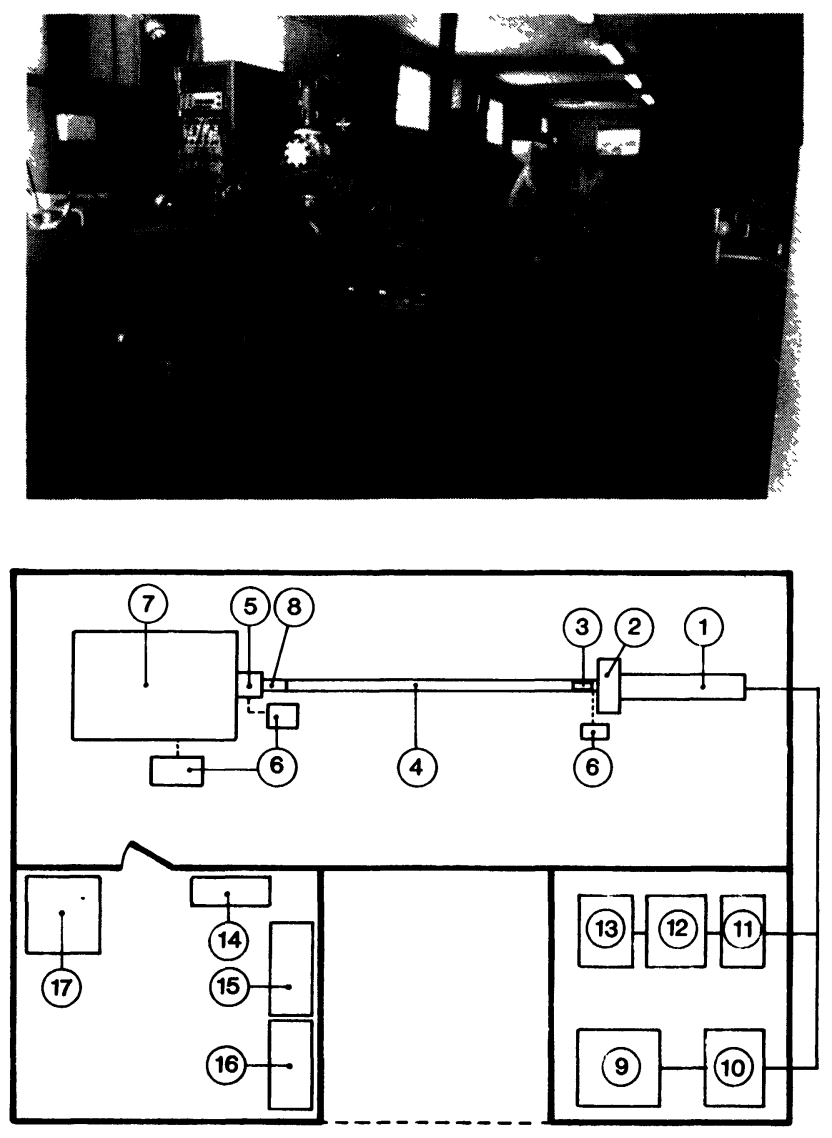

Fig. 1. - Schéma du canon DEMETER (vue de dessus). 1) Réservoir de gaz moteur (longueur $2 \mathrm{~m}, \varnothing$ intérieur $238 \mathrm{~mm}$ ). 2) Vanne à tiroir ( $\varnothing$ d'ouverture $101,6 \mathrm{~mm}$ ). 3) Projectile $(\varnothing 110,38 \mathrm{~mm}$ élancements compris entre 2 et 1). 4) Tube de lancement ( $\varnothing$ intérieur $110,40 \mathrm{~mm}$, longueur $5,83 \mathrm{~m}$ ). 5) Chambre porte-cible (cube de $400 \mathrm{~mm}$ de côté). 6) Pompes à vide (pompes associées à la chambre de détente, à la chambre porte-cible, au tube : débits respectifs $1000 \mathrm{~m}^{3} / \mathrm{h}, 150 \mathrm{~m}^{3} / \mathrm{h}, 16 \mathrm{~m}^{3} / \mathrm{h}$ ). 7) Récupération des éclats $(\varnothing 2 \mathrm{~m}$, longueur $3,3 \mathrm{~m}$ ). 8) Pièce de bouche (isodiamètre avec le tube, longueur $175 \mathrm{~mm}$ ). 9) Compresseur d'air (débit $40 \mathrm{Nm}^{3} / \mathrm{h}$ ). 10) Stockage d'air comprimé (24 bouteilles de 501 à 250 bar maxi.). 11) Stockage d'hélium comprimé (7 bouteilles de 501 à 350 bar maxi.). 12) Surpresseur d'hélium (débit $4 \mathrm{Nm}^{3} / \mathrm{h}$ ). 13) Alimentation en hélium (16 bouteilles de 501 à 200 bar). 14) Pupitre de commande. 15) Baies de mesure. 16) Traitement informatique. 17) Interféromètre " VISAR ».

[Schematic of the gas gun.]

Quatre grands principes ont présidé à la conception du canon :

- Afin d'explorer le domaine élasto-plastique des solides (quelques dizièmes à quelques dizaines de $\mathrm{GPa})$, la gamme de vitesses des projectiles $\left(V_{\mathrm{p}}\right)$ visée s'étend de quelques dizaines de $\mathrm{m} / \mathrm{s}$ à environ $1000 \mathrm{~m} / \mathrm{s}$.

- Pour permettre l'étude des solides hétérogenes ou poreux, la surface sollicitée doit être suffisamment importante : environ $100 \mathrm{~cm}^{2}$.

- Par souci de simplification au niveau de la conception, et afin d'optimiser le parallélisme à l'impact, le canon doit être sans recul.

- Le temps d'exécution d'un essai doit être minimisé et le personnel nécessaire limité à un opérateur et son assistant.

Le modèle mathématique utilisé pour schématiser le canon et calculer ses performances en fonction des dimensions envisagées, est basé sur la méthode des caractéristiques, et n'est qu'un cas particulier de résolution d'un problème communément appelé «problème de Lagrange ». Nous avons largement utilisé les travaux de A. Journeau [1] et W. Heybey [2] pour la partie théorique, et confronté nos prévisions aux solutions semi-empiriques de Seigel [3].

\section{Description du canon.}

3.1 Production des gaz moteurs. - Deux gaz ont été choisis pour couvrir le domaine de vitesses visé : air et hélium. Les caractéristiques de compression et de stockage sont respectivement de $40 \mathrm{Nm}^{3} / \mathrm{h}, 1200 \mathrm{l}$ à 250 bar pour l'air et de $4 \mathrm{Nm}^{3} / \mathrm{h}, 350 \mathrm{l}$ à $350 \mathrm{bar}$ pour l'hélium.

3.2 RÉSERVOIR DE GAZ MOTEUR. - Son volume intérieur est de $95 \mathrm{l}$. Il a été calculé pour une pression motrice maximale de 250 bar. Sa longueur intérieure ( $2 \mathrm{~m}$ ) est telle que les ondes de détente qui prennent naissance lors de la libération du gaz moteur et se réfléchissent sur le fond du réservoir ne puissent pas rattrapper le projectile avant l'impact sur la cible.

3.3 Dispositif DE libÉRATION DU GAZ. - C'est une solution originale qui a été adoptée sous la forme d'une vanne électropneumatique à tiroir. Telle qu'elle se présente aujourd'hui, la vanne modèle $498-4 \mathrm{NC}$ CEG est le fruit d'une coopération entre la Société SECO-DYN [4] et le Centre d'Etudes de Gramat. Son diamètre d'ouverture est de $101,6 \mathrm{~mm}$ et le temps d'ouverture totale est de $600 \mu$ s environ. Elle est entièrement équipée de joints toriques qui assurent, de part et d'autres du tiroir, l'étanchéité entre un vide de $10^{-2}$ torr et une pression maximale d'air ou d'hélium de 250 bar.

La vanne est fixée sur le réservoir. Pour la mise en place d'un projectile dans le tube de lancement, un vérin translate de $260 \mathrm{~mm}$ vers l'arrière l'ensemble vanne-réservoir. Avant le tir, cet ensemble pesant $1100 \mathrm{~kg}$ vient s'ajuster dans le tube de lancement, puis est rendu solidaire du massif support pour annuler le recul lors du tir. Deux boulons en acier de $54 \mathrm{~mm}$ de diamètre sont utilisés à cet effet.

3.4 TUbe DE LANCEMENT. - C'est un fût de canon de calibre $105 \mathrm{~mm}$ qui a été choisi. Il est très largement surdimensionné en épaisseur pour les pressions de service appliquées et sa conception "autoporteur» 
d'origine garantit une rectitude parfaite. Initialement rainuré, il a été réalésé en fût lisse de $110,4 \mathrm{~mm}$ de diamètre. Sa longueur de 5,86 $\mathrm{m}$ permet d'approcher de façon satisfaisante les vitesses maximales des projectiles (Fig. 2), notamment dans le cas de l'air.

A l'extrémité du tube de lancement se trouve une

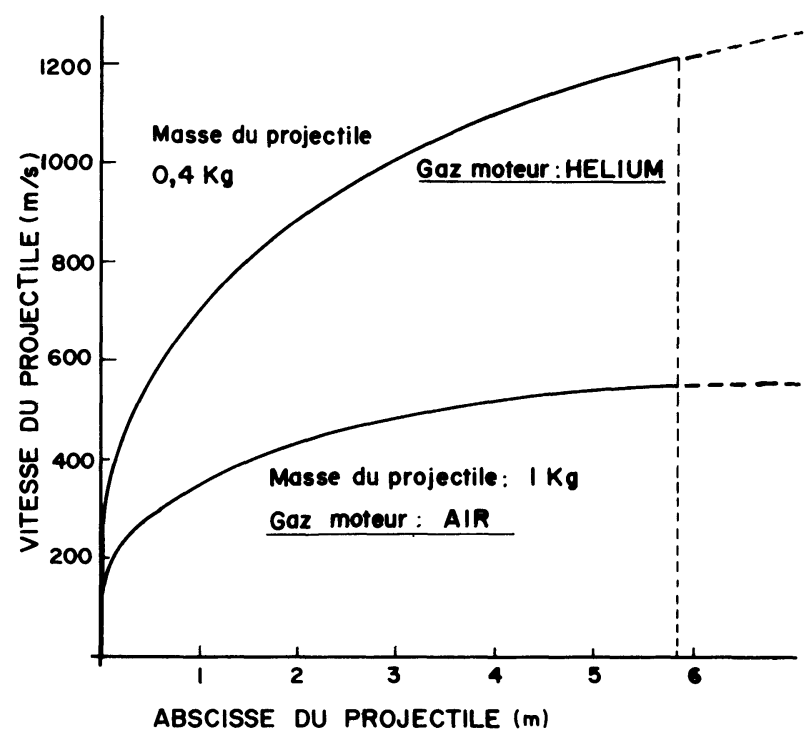

Fig. 2. - Vitesse du projectile dans le tube de lancement. Pression motrice $=250$ bar. Calculs analytiques.

[Velocity of projectile in the gun barrel. Actuating pressure : 250 bar. Analytical calculations.]

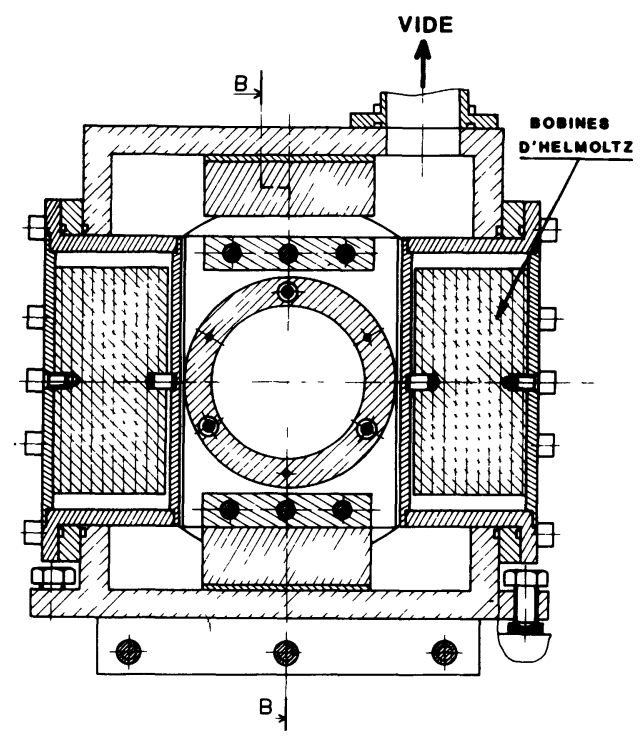

A) coupe aA avec oemerateun de champ magnetioue

Fig. 3a. - Chambre porte-cible. Version mesures électromagnétiques.

[Target chamber-electromagnetic velocity measurements.] pièce de bouche qui assure la liaison avec la chambre porte-cible et supporte les deux barrières optiques de mesure de vitesse du projectile. Chaque barrière est composée d'un laser $\mathrm{He}-\mathrm{Ne}$ et d'une photo-diode P.I.N.

3.5 Chambre PORTE-Cible. - La chambre portecible est une structure mécano-soudée représentée sur la figure 3. A l'avant, la liaison avec la pièce de bouche est faite par l'intermédiaire d'un joint plat en caoutchouc qui assure l'étanchéité au vide et l'isolation vis-à-vis des vibrations. A l'arrière, un diaphragme en aluminium isole la chambre dans laquelle règne un vide de $10^{-2}$ torr avant l'impact (voir paragraphe 3.7 ). Il est perforé par les fragments de cible et de projectile.

La cible instrumentée (Fig. 4) est montée dans une couronne porte-cible qui est ensuite fixée sur un tiroir. La distance entre la face avant de la cible et la bouche du canon est de $200 \mathrm{~mm}$. Trois vis placées à $120^{\circ}$ permettent de régler la position de la cible de façon à obtenir le parallélisme cible-impacteur requis. La perpendicularité entre la face avant de la cible et l'axe du tube de lancement est obtenue par alignement optique. La procédure est la suivante : on positionne un faisceau laser de manière à ce qu'il matérialise l'axe du tube de lancement; un miroir, plaqué par dépression sur la face avant de la cible, réfléchit ce faisceau et par action sur les trois vis de réglage précédemment mentionnées, on oriente la cible de manière à faire confondre faisceau incident

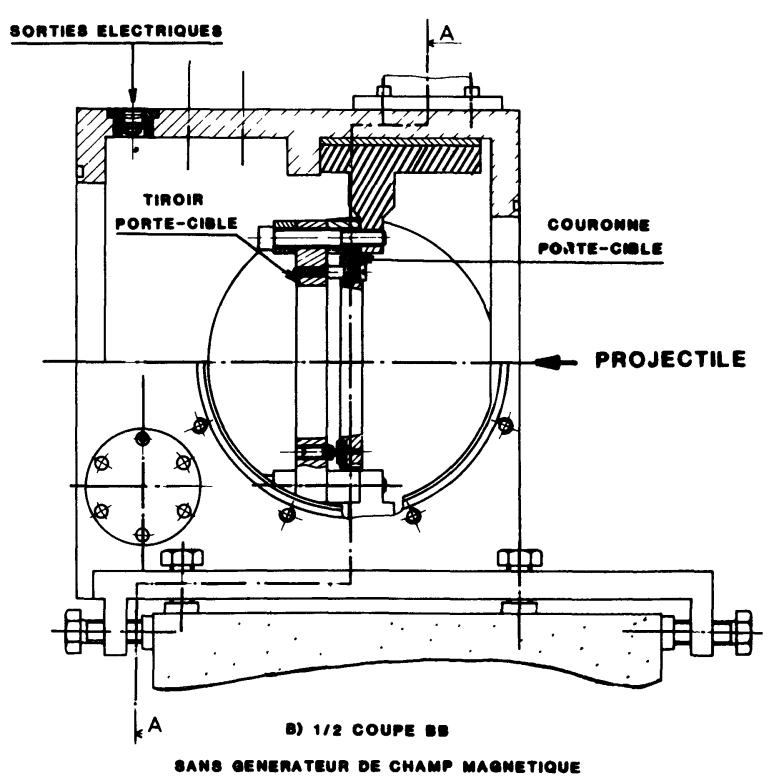

Fig. 3b. - Chambre porte-cible. Version jauges piézorésistives.

[Target chamber-piezoresistive gauges measurements.] 


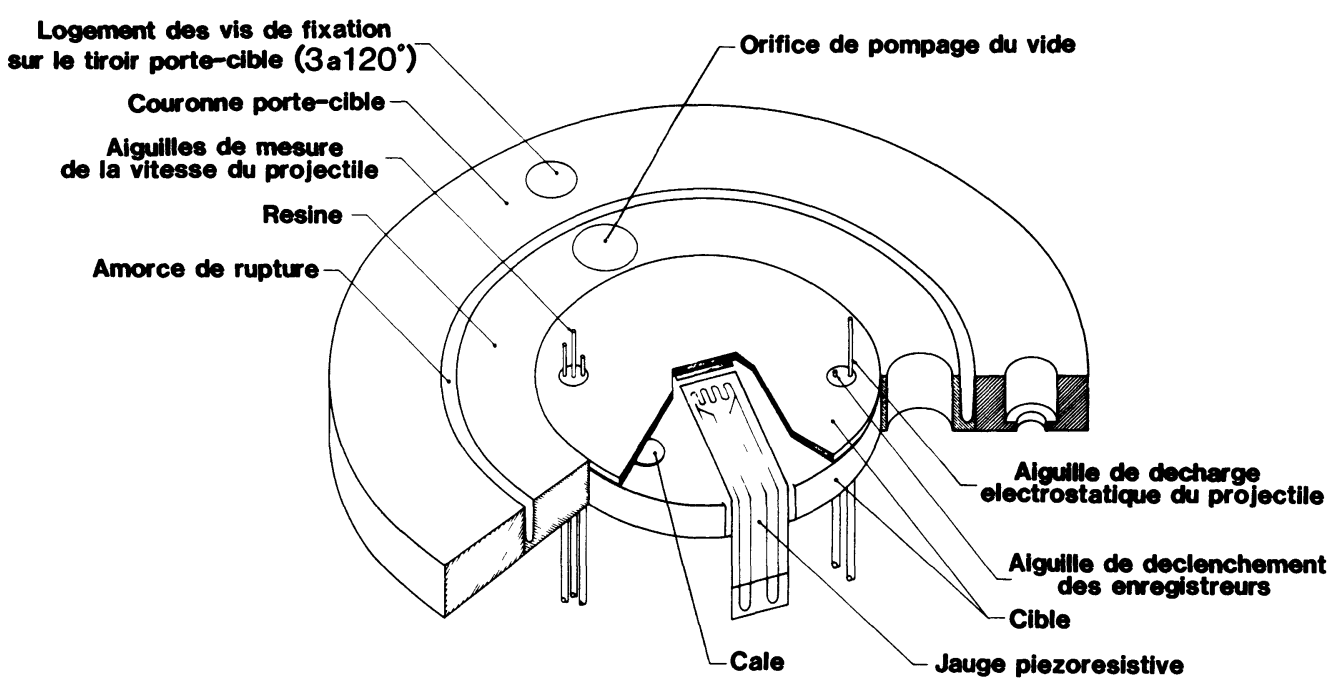

Fig. 4. - Schéma éclaté d'une cible instrumentée de jauges piézorésistives.

[Cut away schematic of a target including piezoresistive gauges.]

et faisceau réfléchi. La chambre porte-cible permet plusieurs types de mesures. Sur la figure 3a, elle est équipée de bobines d'Helmoltz associées aux mesures électro-magnétiques de vitesses matérielles. Pour les mesures de contraintes par jauges piézorésistives (Fig. 3b), on remplacera les caissons contenant les bobines par de simples tapes métalliques. Dans les deux cas, des passages électriques assurent la sortie des signaux. Pour les expérimentations de type optique (interférométrie et ombroscopie), les tapes sont remplacées par des hublots en PMMA.

3.6 DisPositiFs DE DÉTENTE DES GAZ MOTEURS ET DE RÉCUPÉRATION DES ÉCLATS. - Il existe deux possibilités suivant les besoins expérimentaux : dans le cas où les fragments de cible doivent être analysés, la chambre porte-cible est liée à une chambre de détente d'un volume intérieur de $10 \mathrm{~m}^{3}$ équipée d'un caisson de récupération; dans le cas contraire, un tube de focalisation télescopique évacue les fragments vers une fosse à sable à l'extérieur du laboratoire.

\subsection{GROUPES DE POMPAGE ET DE CONTRÔLE DU VIDE. -} Pour éviter de pousser devant le projectile un coussin de gaz parasite, l'ensemble du tube de lancement et de la chambre porte-cible est mis sous un vide de $10^{-2}$ torr au moyen de deux groupes. L'un est connecté à la chambre porte-cible $\left(150 \mathrm{~m}^{3} / \mathrm{h}\right)$ et l'autre à l'arrière du tube de lancement $\left(16 \mathrm{~m}^{3} / \mathrm{h}\right)$. La différence de volume de ces deux chambres séparées par le projectile et une temporisation adéquate des déclenchements des deux groupes, assurent le maintien en place du projectile avant tir. Un troisième groupe $\left(1000 \mathrm{~m}^{3} / \mathrm{h}\right)$ est utilisé pour amener la chambre de détente à $10^{-1}$ torr.
3.8 Pupitre De COMmande. - Il est situé dans une salle adjacente au laboratoire et permet de télécommander la séquence de tir. Après avoir mis en place la cible, le diaphragme de la chambre portecible, le projectile et l'ensemble vanne-réservoir, l'opérateur commande depuis le pupitre les opérations suivantes : mise en route des dispositifs de sécurité, déclenchement des groupes de pompage. remplissage du réservoir, armement de la vanne ultrarapide et enfin déclenchement du tir puis remise à pression atmosphérique de la chambre de détente.

Outre ces commandes, on trouve également sur le pupitre un synoptique de l'installation avec voyants lumineux et indicateurs de pressions.

3.9 Projectiles. - Deux types de sabots sont principalement utilisés. Les sabots "standards " dont la masse peut être choisie entre $1,4 \mathrm{~kg}$ et $6 \mathrm{~kg}$ sont réservés aux vitesses faibles et moyennes avec propulsion à l'air (Fig. 5a). Les sabots " légers» dont la masse est de $0,4 \mathrm{~kg}$ permettent d'atteindre les vitesses les plus élevées dans le cas de la propulsion à l'hélium ou bien à l'air à haute pression (Fig. 5b). Ces deux types de sabots sont en aluminium et les segments en téflon.

\section{Performances.}

Les résultats obtenus se sont avérés très proches des prévisions pour les diverses caractéristiques énumérées ci-dessous.

4.1 VitesSe du PROJECTILE À L'IMPACT. - La vitesse à l'impact programmée peut être obtenue en jouant 

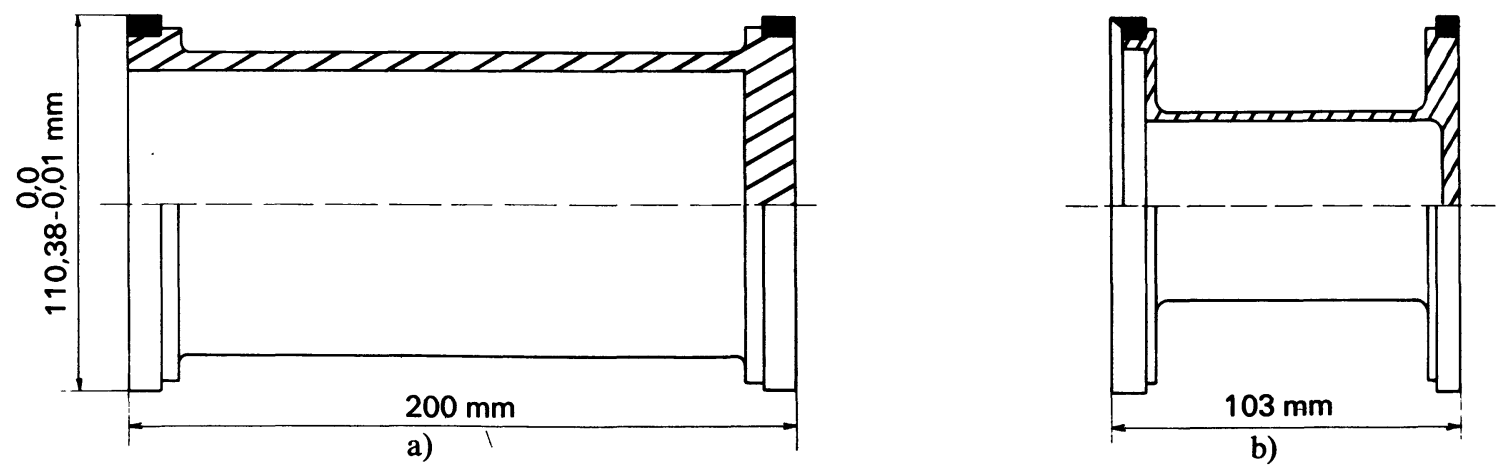

Fig. 5. - Projectiles pour sollicitation de choc plan. a) « Projectile standard ». b) " Projectile léger ».

[Projectiles for plane shock wave experiments.]

sur trois paramètres : la nature du gaz moteur, sa pression et la masse du projectile.

Le volume du réservoir étant fixé, ces trois paramètres sont représentés par le rapport $G / M_{\mathrm{p}}$ entre la masse du gaz moteur $G$ et la masse du projectile $M_{\mathrm{p}}$. L'étalonnage du canon a consisté à mesurer systématiquement les vitesses de projectiles associées à des valeurs $G / M_{\mathrm{p}}$ différentes.

Pour l'air comme gaz moteur, la gamme des $G / M_{\mathrm{p}}$ qui a été explorée est comprise entre 0,2 et 40 . Ces bornes correspondent à des couples pression motricemasse de projectile de 10 bar- $6 \mathrm{~kg}$ pour $G / M_{\mathrm{p}}=0,2$ et 200 bar- $0,6 \mathrm{~kg}$ pour $G / M_{\mathrm{p}}=40$.

Dans le cas de l'hélium, la gamme de $G / M_{\mathrm{p}}$ est comprise entre 1 et 10 , ce qui correspond à des couples pression motrice-masse de projectile de 30 bar- $0,5 \mathrm{~kg}$ et 230 bar- $0,4 \mathrm{~kg}$.

La vitesse du projectile $V_{\mathrm{p}}$ est mesurée au moyen de deux méthodes. D'une part, les barrières optiques situées à l'extrémité du tube de lancement, et d'autre part, des aiguilles coaxiales à court-circuit solidaires de la cible (Fig. 4). C'est le contact entre les extrémités d'aiguilles polarisées et la face avant du sabot qui engendre les impulsions de chronométrie.

Ces deux méthodes ont une précision identique et égale à $1 \%$. Les résultats de 110 essais représentés sur la figure 6 prouvent la bonne concordance de ces deux types de mesures et montrent que l'on dispose, pour les domaines de $G / M_{p}$ détaillés précédemment, d'une gamme de vitesses continûment programmable de $100 \mathrm{~m} / \mathrm{s}$ à $660 \mathrm{~m} / \mathrm{s}$ pour l'air et de $560 \mathrm{~m} / \mathrm{s}$ à $1150 \mathrm{~m} / \mathrm{s}$ pour l'hélium. La répétitivité des vitesses programmées, primordiale pour certaines applications, est inférieure à $1 \%$ pour des conditions expérimentales rigoureusement identiques.

4.2 OBLIQUité À L'IMPACT. - Elle résulte de plusieurs imprécisions expérimentales : défauts de positionnement de la cible, défaut de perpendicularité entre la face avant de l'impacteur et ses génératrices définies par les segments du sabot, ou encore déviation de la trajectoire du projectile. L'angle d'obliquité à l'impact $(\alpha)$ est mesuré au moyen de sondes à court-circuit et pour les essais couramment réalisés : $\alpha<10^{-3} \mathrm{rd}$.

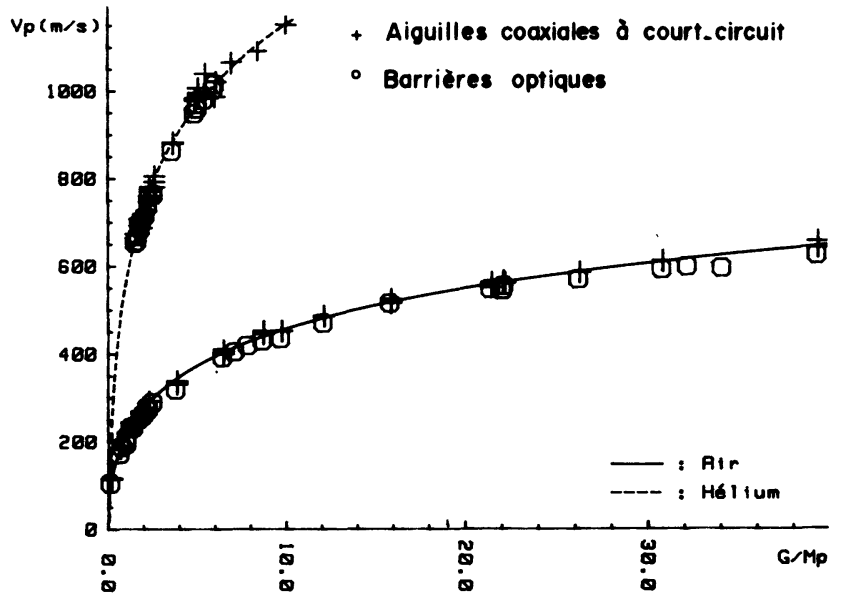

Fig. 6. - Vitesse du projectile mesurée à l'impact en fonction du rapport entre la masse de gaz moteur et la masse de projectile.

[Measured projectile velocity at impact versus the ratio between masses of actuating gases and projectile.]

\section{Comparaison des performances du canon DEMETER et de celles de canons similaires.}

Tous les grands laboratoires de physique des chocs sont dotés de canons : canons à gaz à un étage tels que DEMETER, canons à poudre à un étage, canons à deux étages (poudre, gaz léger), ou bien canons à rails ou canons électriques. Afin de comparer les performances du canon DEMETER à celles d'installations similaires, nous avons essayé de recenser sur le tableau I, les canons à gaz à un étage en éliminant ceux qui ne sont pas utilisés à des fins identiques à celles du canon DEMETER : l'étude du comportement sous choc de la matière. Ont par exemple été éliminées de notre recensement, les installations destinées aux études d'aérobalistique (vol libre du projectile en bout du tube de lancement) telles celles du Harry Diamond Laboratory ou d'Eglin Air Force Base. Ont également été éliminés les canons utilisés pour reproduire des chutes libres 
Tableau I. - Caractéristiques comparées de canons à gaz à un étage et fût lisse.

\begin{tabular}{|c|c|c|c|c|c|c|c|c|c|}
\hline \multirow{3}{*}{ Laboratoires } & \multirow{3}{*}{$\begin{array}{l}\text { Natio- } \\
\text { nalité }\end{array}$} & \multicolumn{2}{|c|}{ Tube de lancement } & \multirow{2}{*}{$\begin{array}{l}\text { Masses de } \\
\text { projectiles } \\
M_{\mathrm{p}}\end{array}$} & \multirow{2}{*}{$\begin{array}{c}\text { Pression } \\
\text { motrice } \\
\text { maximale } P_{0}\end{array}$} & \multirow{2}{*}{$\begin{array}{c}\text { Dispositif } \\
\text { de libération } \\
\text { du gaz moteur }\end{array}$} & \multirow{2}{*}{$\begin{array}{c}\text { Vitesse } \\
\text { maximale } \\
\text { a la bouche }\end{array}$} & \multirow{2}{*}{$\begin{array}{l}\text { Obliquité } \\
\text { à l'impact }\end{array}$} & \multirow{2}{*}{ Références } \\
\hline & & $\begin{array}{l}\text { diamètre } \\
\text { intérieur }\end{array}$ & longueur & & & & & & \\
\hline & & $\mathrm{mm}$ & $\mathrm{m}$ & $\mathrm{kg}$ & bar & & $\mathrm{m} / \mathrm{s}$ & mrd & \\
\hline $\begin{array}{l}\text { Commissariat à } \\
\text { l'Energie } \\
\text { Atomique }\end{array}$ & $\mathrm{F}$ & 98 & 4 & 2 à 10 & $1000\left(\mathrm{~N}_{2}\right)$ & «Wrap-around » & $\begin{array}{c}450 \\
\left(P_{0}=200 \mathrm{~b}\right) \\
\left(M_{\mathrm{p}}=2,9 \mathrm{~kg}\right)\end{array}$ & & {$[8]$} \\
\hline $\begin{array}{l}\text { Institut Franco- } \\
\text { Allemand de } \\
\text { Recherches de } \\
\text { Saint-Louis }\end{array}$ & F/RFA & 90 & & & $\begin{array}{r}350 \\
\text { (Air-He) }\end{array}$ & $\begin{array}{c}1 \\
\text { diaphragme }\end{array}$ & 1000 & 1 & {$[9,10]$} \\
\hline $\begin{array}{l}\text { Centre Etudes de } \\
\text { Gramat }\end{array}$ & $\mathrm{F}$ & 110,4 & 5,86 & 0,4 à 6 & $\begin{array}{r}250 \\
\text { (Air-He) }\end{array}$ & $\begin{array}{c}\text { vanne } \\
\text { ultra-rapide }\end{array}$ & 1150 & 1 & \\
\hline $\begin{array}{l}\text { Institut } \\
\text { Ernst. Mach. }\end{array}$ & RFA & $\begin{array}{r}50 \\
100\end{array}$ & & & & & $\begin{array}{l}500 \\
500\end{array}$ & & [11] \\
\hline $\begin{array}{l}\text { Department of } \\
\text { Physics Washing- } \\
\text { ton State Univ. }\end{array}$ & USA & 101,6 & 12,25 & $\begin{array}{l}0,6 \\
1,10\end{array}$ & $\begin{array}{l}413 \\
206\end{array}$ & $\begin{array}{l}\text { double-diaphragme } \\
\text { "Wrap-around " }\end{array}$ & $\begin{array}{r}1500 \\
900\end{array}$ & 0,5 & [5] \\
\hline $\begin{array}{l}\text { Los Alamos } \\
\text { National } \\
\text { Laboratory }\end{array}$ & USA & 70 & 7,6 & & $\begin{array}{r}430 \\
1030\end{array}$ & $\begin{array}{l}\text { "Wrap-around " } \\
\text { double-diaphragme }\end{array}$ & $\begin{array}{l}1000 \\
1600\end{array}$ & & [12] \\
\hline $\begin{array}{l}\text { Lawrence Liver- } \\
\text { more National } \\
\text { Laboratory }\end{array}$ & USA & 101,6 & 18,3 & & $\begin{array}{r}210 \\
\text { (Air-He) }\end{array}$ & «Wrap-around" & 500 & & [13] \\
\hline $\begin{array}{l}\text { Sandia National } \\
\text { Laboratories }\end{array}$ & USA & $\begin{array}{r}63 \\
100\end{array}$ & $\begin{array}{l}25 \\
7,32\end{array}$ & $\begin{array}{c}0,25 \text { à } 1 \\
0,9\end{array}$ & $\begin{array}{c}350 \\
(\text { Air- } \mathrm{He}) \\
378 \\
\left(\text { Air, } \mathrm{N}_{2}, \mathrm{He}\right)\end{array}$ & $\begin{array}{l}\text { double-diaphragme } \\
\text { vanne ultra-rapide }\end{array}$ & $\begin{array}{l}1600 \\
1000\end{array}$ & $\begin{array}{c}0,2 \\
0,2 \text { à } 0,5\end{array}$ & $\begin{array}{c}{[14,16]} \\
{[17]}\end{array}$ \\
\hline Brown University & USA & $\begin{array}{r}63,5 \\
101,6\end{array}$ & $\begin{array}{l}2,5 \\
3,4\end{array}$ & & & $\begin{array}{l}300 \\
500\end{array}$ & & & {$[18]$} \\
\hline S.R.I. International & USA & $\begin{array}{r}63,5 \\
101,6\end{array}$ & $\begin{array}{r}2 \\
10\end{array}$ & $\begin{array}{r}0,5 \text { à } 1 \\
0,5 \text { à } 1 \\
1 \text { à } 4\end{array}$ & $\begin{array}{l}420 \\
420\end{array}$ & $\begin{array}{l}\text { "Wrap-around" } \\
\text { double diaphragme } \\
\text { "Wrap-around" }\end{array}$ & $\begin{array}{r}600 \\
1500 \\
1200\end{array}$ & 0,5 & $\begin{array}{l}{[19]} \\
{[20]}\end{array}$ \\
\hline $\begin{array}{l}\text { University of } \\
\text { Dayton Research } \\
\text { Institute }\end{array}$ & USA & $\begin{array}{r}51 \\
89 \\
178\end{array}$ & $\begin{array}{l}7,3 \\
6 \\
9\end{array}$ & $\begin{array}{l}0,3 \\
0,7 \\
4,5\end{array}$ & $\begin{array}{r}\text { (Air-He) } \\
\text { (air) } \\
\text { (air) }\end{array}$ & & $\begin{array}{l}500 \\
300 \\
270\end{array}$ & & {$[21]$} \\
\hline $\begin{array}{l}\text { Boeing Shock } \\
\text { Physics Labo. }\end{array}$ & USA & 64 & & & $\begin{array}{r}420 \\
(\mathrm{He})\end{array}$ & & 1400 & & {$[22]$} \\
\hline Dynasen & USA & $\begin{array}{r}64 \\
64 \\
128\end{array}$ & $\begin{array}{l}2,5 \\
6,2 \\
6,2\end{array}$ & $\begin{array}{l}0,1 \text { à } 0,3 \\
0,1 \text { à } 0,3\end{array}$ & $\begin{array}{r}140 \\
\text { (Air-He) }\end{array}$ & & $\begin{array}{r}500 \\
1100 \\
600\end{array}$ & & [13] \\
\hline
\end{tabular}

de structures tels que les canons du CEA/CESTA. Enfin, nous n'avons pas retenu les installations pour lesquelles nous n'avions que des données trop partielles.

Le tableau I permet de dégager les observations suivantes :

- La grande majorité des canons se trouve dans des laboratoires américains, les lanceurs de l'Union Soviétique n'ayant pu être recensés par manque de données.

- Peu de canons utilisent un système de vanne ultra-rapide et sont, par contre, équipés de dispositifs à diaphragmes ou encore de "wrap-around " (réservoir et orifices annulaires autour du tube de lance- 
ment, le projectile assurant l'étanchéité avant d'être poussé en avant lors du déclenchement du tir). Seul le canon de $100 \mathrm{~mm}$ de Sandia National Laboratories est équipé d'une vanne ultra-rapide; comme le canon DEMETER, il permet d'atteindre des vitesses de $1000 \mathrm{~m} / \mathrm{s}$ mais doit utiliser pour ce faire une pression motrice de 378 bar contre 250 bar pour DEMETER (les longueurs de tube de lancement étant, par ailleurs, voisines).

Les canons qui utilisent des dispositifs à diaphragmes ou un "wrap-around " avec des pressions motrices comparables à celles de notre canon (250 bar) ne permettent pas des vitesses supérieures (cf. canon de $90 \mathrm{~mm}$ de l'ISL); pour atteindre, en version un étage des vitesses de $1500 \mathrm{~m} / \mathrm{s}$, il faut utiliser des pressions motrices de l'ordre de 400 bar (cf. canon de 101,6 mm de l'Université de Washington). Le choix d'une vanne à ouverture ultra-rapide apparaît donc judicieux car, sans altérer les performances du lanceur, la vanne permet d'une part, de minimiser le temps de mise en ouvre par rapport aux diaphragmes et d'autre part, autorise des sabots légers par rapport à un système " wrap-around " pour lequel le projectile doit résister à la pression motrice appliquée latéralement avant le déclenchement du tir.

- En ce qui concerne l'obliquité à l'impact, les comparaisons sont difficiles car il existe une différence entre les résultats obtenus dans une expérimentation où le parallélisme à l'impact est primordial (mesure de fronts de chocs quasi instantanés) et les essais " courants " où les mesures effectuées ne sont pas affectées par un léger défaut de parallélisme. Les performances annoncées pour le canon DEMETER concernent un essai " courant ".

Il faut noter enfin que la conception de la plupart des canons américains cités dans le tableau I dérive d'un prototype construit en 1970 par Fowles et al. [5] et ont été en général réalisés par des entreprises spécialisées dans ce type d'appareils tel que U.R.D.C. [6] ou G.M. [7]. Contrairement, les canons européens sont plutôt conçus par les utilisateurs eux-mêmes et les technologies adoptées sont davantage diversifiées.

\section{Chaînes de mesure implantées sur le canon DEMETER.}

D'un point de vue macroscopique, une onde de choc induit dans un solide une séquence de phénomènes précis et ordonnés mais évoluant rapidement : le front de choc peut atteindre son amplitude maximale en quelques nanosecondes et un profil d'onde courant n'excède pas quelques microsecondes. C'est pendant cette durée, alors que la matière atteint des états thermodynamiques difficiles ou impossibles à produire par d'autres moyens, que les phénomènes physiques intéressants doivent être examinés. Pour ce faire, diverses chaînes de mesures ont été implantées sur le canon DEMETER.
6.1 Mesures DE CONTRAintes. - Elles mettent en œuvre des jauges piézorésistives qui, insérées dans un échantillon mesurent directement la contrainte appliquée normalement à leur plan.

Des jauges en manganin et en carbone ont été étalonnées au moyen du canon DEMETER entre 0 et $40 \mathrm{GPa}$ [23-25], puis utilisées dans diverses études du comportement élastoplastique des solides sous choc. Les techniques d'étalonnage utilisées sont identiques à celles mises au point par les précurseurs des mesures piézorésistives aux U.S.A. [26] et en Grande Bretagne [27]. La contrainte mesurée peut être normale, les jauges sont alors placées dans un plan parallèle au front de choc (Fig. 4) ou dans plusieurs plans consécutifs (analyse lagrangienne [28]); elle peut également être latérale, dans un plan perpendiculaire au front de choc [29].

La séquence type d'une expérimentation mettant en œuvre des jauges piézorésistives se déroule de la façon suivante : le passage du projectile devant la deuxième barrière optique située à la bouche du canon (cf. § 3.4) déclenche les alimentations impulsionnelles des ponts de Wheatstone associés aux jauges. Puis, quelques microsecondes avant l'impact, le sabot rencontre les aiguilles coaxiales à court-circuit solidaires de la cible.

Ces contacts électriques permettent d'une part, de contrôler la mesure électro-optique, et d'autre part, de déclencher les enregistreurs : analyseurs de phénomènes transitoires Thomson TSN 693 ayant un pas d'échantillonnage de $10 \mathrm{~ns}$. La transformation du signal électrique en profil de contrainte est réalisée avec un mini-ordinateur HP $9845 \mathrm{~B}$. La figure 7a montre un exemple de signal ainsi transformé. Il s'agit du profil de contrainte longitudinale recueilli au sein d'un échantillon de tungstène, à une profondeur de $5 \mathrm{~mm}$. La sollicitation était provoquée par un impacteur également en tungstène propulsé à $612 \mathrm{~m} / \mathrm{s}$. Les jauges utilisées sont deux jauges en manganin HBM de $50 \Omega$ d'impédance [24] placés dans le même plan de mesure.

6.2 Mesures De vitesses MAtérielles. - Par jauges électromagnétiques : l'échantillon est placé dans un champ magnétique uniforme généré par les bobines d'Helmoltz représentées sur la figure 3 . La jauge est une simple boucle de cuivre non alimentée insérée dans l'échantillon à la manière d'une jauge piézorésistive. Elle délivre un courant électrique directement proportionnel à la vitesse matérielle, à l'intensité du champ et à la géométrie de la boucle [30]. Dans la séquence type d'un tel essai, une phase de mise sous tension des bobines d'Helmoltz et de contrôle de l'intensité du champ magnétique conditionne le déclenchement du tir. Les signaux sont enregistrés et traités avec les mêmes chaînes numériques que les signaux piézorésistifs. Un exemple de profil de vitesse matérielle est donné sur la figure $7 \mathrm{~b}$. La jauge utilisée est de fabrication CEG [30], son 


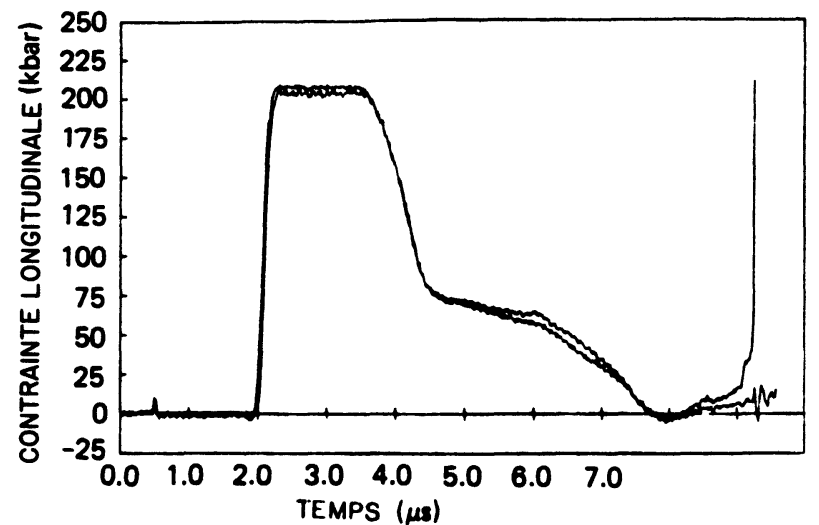

a)

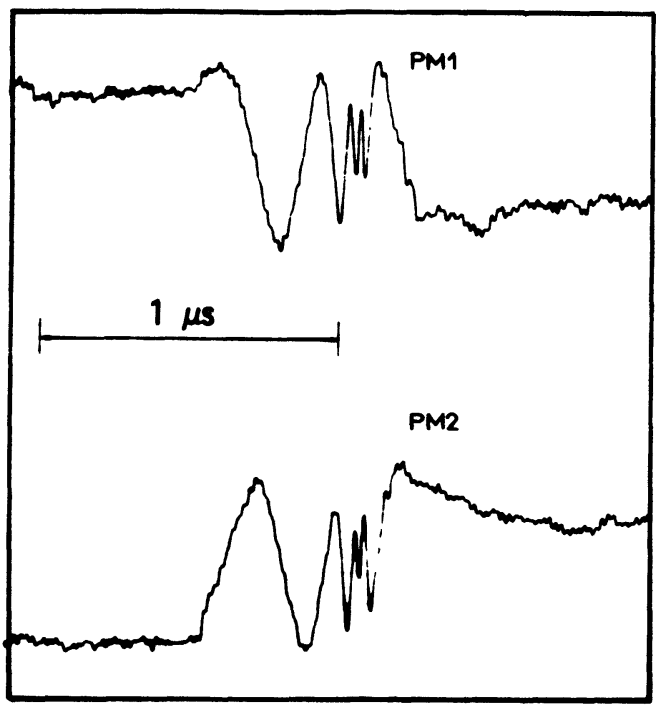

c)

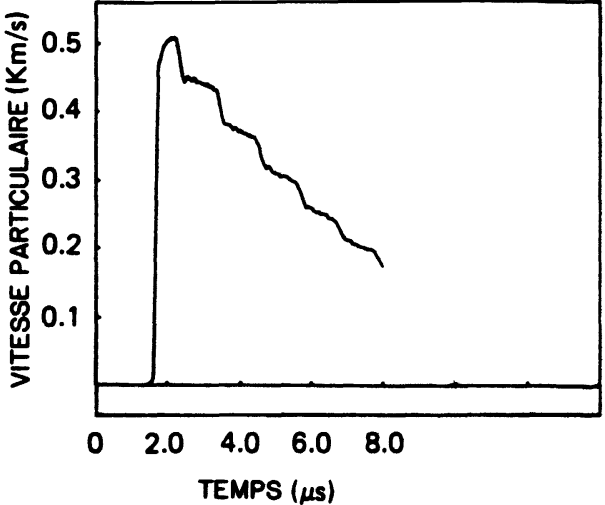

b)

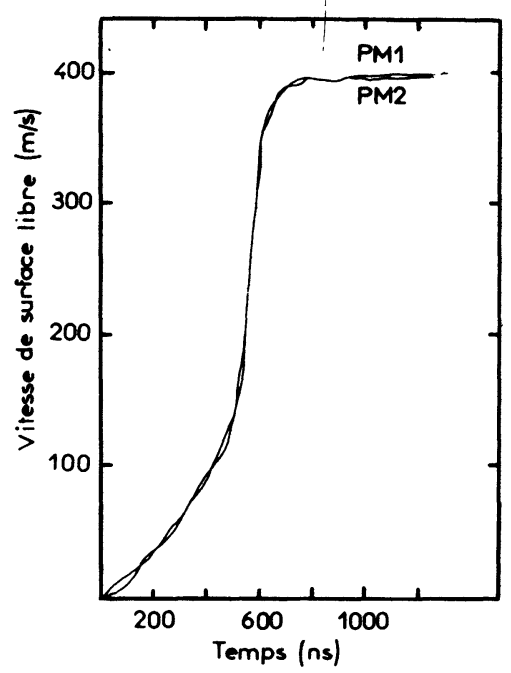

d)

Fig. 7. - Signaux délivrés par les chaînes de mesures implantées sur le canon DEMETER. a) Profil de contrainte dans un échantillon de tungstène ( 2 jauges en manganin dans le même plan de mesure). b) Profil de vitesse matérielle dans un échantillon d'araldite (jauge électromagnétique dans un champ magnétique de 550 Gauss). c) Signaux interférométriques "VISAR » résultant de la mise en vitesse de la surface libre d'un échantillon de tungstène. d) Profil de vitesse obtenue après traitement des signaux interférométriques représentés sur la figure $7 \mathrm{c}$.

[Profiles from diagnostic facilities associated to the gas gun.]

épaisseur totale est de $85 \mu \mathrm{m}$ et la longueur de l'élément actif est de $10 \mathrm{~mm}$. Elle est insérée dans un échantillon d'araldite à une profondeur de $5 \mathrm{~mm}$. L'impacteur est un disque d'alumine propulsé à $573 \mathrm{~m} / \mathrm{s}$.

Le signal montre une sollicitation incidente de $500 \mathrm{~m} / \mathrm{s}$ suivie par des palliers de décompression successifs qui résultent des réflexions d'ondes entre la face avant de l'échantillon et la face arrière libre de l'impacteur.

- Par interférométrie : la face arrière de l'échantillon, libre ou accolée à une fenêtre transparente, est illuminée par un faisceau laser. La variation de la longueur d'onde réfléchie (effet Doppler) est analysée au moyen d'un interféromètre de type " VISAR " [31] et [32]. Le défilement des franges est détecté par des tubes photomultiplicateurs déclenchés lors du passage du projectile devant la deuxième barrière optique. Les signaux délivrés par les photomultiplicateurs sont mesurés au moyen d'analyseurs Thomson TSN 694 avec un pas d'échantillonnage de 2 nanosecondes adapté à la résolution du "VISAR " (quelques nanosecondes). La figure $7 \mathrm{c}$ représente les signaux interférométriques dans le cas d'une mesure sur la face arrière libre d'un échantillon de tungstène sollicité par une onde de choc de 14,1 GPa d'intensité (le "VISAR " délivre deux signaux en quadrature afin de lever d'éventuelles indéterminations en cas 
de changement de signe du gradient de vitesse). La transformation de ces signaux interférométriques en profils de vitesse (Fig. 7d) est réalisée au moyen du calculateur déjà mentionné.

6.3 OMBroscopIE. - Pour certaines applications telles que les tests de plasticité dynamique de Taylor [33], il est nécessaire de visualiser le phénomène d'impact en cinématographie ultra-rapide. Nous utilisons à cet effet une chronoloupe à 16 diodes de type Crantz Schardin "AID » [34] permettant une cadence de prises de vues maximum de $10^{7}$ images par seconde. La chronoloupe est déclenchée par le passage devant la deuxième barrière électro-optique évoquée précédemment.

\section{Conclusions.}

Les performances du canon DEMETER permettent d'explorer aisément les domaines de comportement élasto-plastique de la plupart des matériaux puisque la vitesse du projectile est continûment programmable entre $100 \mathrm{~m} / \mathrm{s}$ et $1150 \mathrm{~m} / \mathrm{s}$ avec une reproductibilité de $1 \%$; à titre d'exemple, on peut solliciter le PMMA jusqu'à $2 \mathrm{GPa}$, le cuivre jusqu'à $10 \mathrm{GPa}$ et le tungstène jusqu'à $40 \mathrm{GPa}$.

La limite inférieure de $100 \mathrm{~m} / \mathrm{s}$ n'est imposée que par le critère de reproductibilité en raison de la pente excessive de la courbe de prédiction $V_{\mathrm{p}}\left(G / M_{\mathrm{p}}\right)$ à proximité de l'origine. Ces performances, comparées à celles de canons similaires utilisant, outre-atlantique, des dispositifs de libération des gaz moteurs plus conventionnels tels que les diaphragmes simples ou doubles ou encore les "wrap-around", montrent que l'option vanne à tiroir choisie ne diminue pas les performances en vitesse. Elle permet en outre d'obtenir une meilleure répétitivité et un temps de mise en œuvre plus réduit puisque on effectue couramment deux essais par jour lors des campagnes d'expérimentations.

Les chaînes de mesures implantées sur le canon et reliées à un calculateur permettent d'obtenir aisément les signaux de contrainte ou de vitesse matérielle avec une résolution temporelle de quelques dizaines de nanosecondes pour les essais avec jauges, et de quelques nanosecondes pour les mesures interférométriques.

Il convient d'ajouter aux chaînes décrites dans cette publication d'autres possibilités que nous énumérerons simplement : mesures par quartz (vitesses d'ondes et contrainte transmise), mesures par jauges piézoélectriques PVF2, dispositif escamotable de chauffage de cible, qui contribuent également à faire de l'ensemble DEMETER un outil puissant et sophistiqué d'investigation en physique du solide sous choc.

\section{Bibliographie}

[1] Journeau, A., Etude théorique du canon à gaz léger, Mémorial de l'Artillerie Française (1963).

[2] HEBEY, W., A solution of Lagrange's problem of interior ballistics by means of its characteristics lines. Rapport US Naval Ordnance Laboratory $\mathrm{n}^{\circ}$ NOLM 10819 (1950).

[3] SeIGel, The theory of high speed guns, United States Naval Ordnance Laboratory (1965). Office of Naval Res, Dept of the Navy Washington Dc.

[4] Vanne 498-4NC-CEG Société SECO-DYN, San Lorenzo street, Pomona, California, USA.

[5] Fowles, G. R., Duvall, G. E., Asay, J., Bellamy, P., Feistmann, F., Grady, D., Michaels, T., MitCHELL, R., Rev. Sci. Instrum. 41 (7) (1970).

[6] Utah Research and Development Compagny, 7915 South, 1530 West, West Jordan Utah 84 084, USA.

[7] Delco, General Motors Corporation, Santa Barbara Operations, 6767 Hollister Avenue, Goleta, California 93117 , USA.

[8] Lezaud, J. M., Delbar, P., Invernizzi, M., Huz, R., Lanceur à gaz " hélène ", calibre $98 \mathrm{~mm}$, Rapport technique CEA, Centre d'Etudes de Vaujours (non publié).

[9] Samirant, M., Mise au point d'un canon à air comprimé de $90 \mathrm{~mm}$ lançant des projectiles ultra-légers. Notice ISL $N$ 11/72.
[10] BAUER, F., ISL Shock wave facilities, AIP Conference Proceedings, Shock waves in condensed mater $\mathrm{n}^{\circ} 78$, p. $674-679$ (1981).

[11] Stilp, A. J., Hohler, V., Schneider, E., Tham, R. HÜLSEWIG, M., KuSCHER, G., JuNCKERMANN, W., Impact facilities at the Ernst Mach Institute, AIP Conference Proceedings, Shock waves in condensed matter, $\mathrm{n}^{\circ} 78$, p. $711-712$ (1981).

[12] Vorthman, J. E., Facilities for the study of shock induced decomposition of high explosives, AIP Conference Proceedings, Shock waves in condensed matter, $\mathrm{n}^{\circ} 78$, p. 680-684 (1981).

[13] Fuller, P. W. W., Survey of aeroballistic Launcher and Range facilities. Part. 1, 34th meeting of the aeroballistic Range Association (1983).

[14] Thunborg, S., Jr, Ingram, G. E. and Graham, R. A., Rev. Sci. Instrum. 35 (1964) 11.

[15] InGRAM, G. E., Rev. Sci. Instrum. 36 (1965) 458.

[16] Ingram, G. E. and Graham, R. A., in Fifth Symposium on Detonation, Office of Naval Research, Report ACR-184, August 1980, Edited by S. Jacobs and R. Roberts, p. 369.

[17] Lundergan, C. D., Sandia Coporation Report, No SC-RR, 4421 (1960), unpublished.

[18] Clifton, R. J., Plate impact facility at Brown University, AIP Conference Proceedings, Shock waves in condensed matter $\mathrm{n}^{\circ} 78$, p. 661-662 (1981). 
[19] Gupta, Y. M., Keough, D. D., Walter, D. F., DaO, K. A., Henley, D., UrWeider, A., Rev. Sci. Instrum. 51 (2) (1980).

[20] Murri, W. J., Shock wave facilities at Poulter Laboratory of S.R.I. International AIP Conference Proceedings, Shock waves in condensed matter $\mathrm{n}^{\mathrm{o}} 78$, p. 652-656 (1981).

[21] BLess, S. J., Impact physics facilities at the University of Dayton Research Institute, AIP Conference Proceedings, Shock waves in condensed matter no 78, p. 668-673.

[22] SChmidT, R. M., Boeing Shock Physics Laboratory, AIP Conference Proceedings, Shock waves in condensed matter $\mathrm{n}^{\circ} 78$, p. 634-638.

[23] Perez, M., Chartagnac, P., Shock loading and unloading behaviour of carbon piezoresistive gauges up to $5 \mathrm{GPa}$, Rev. Sci. Instrum. $51 \mathrm{n}^{\circ} 7$ (1980).

[24] Perez, M., Contribution à l'étude du comportement sous choc des jauges piézorésistives jusqu'à $100 \mathrm{~Kb}$, Thèse Docteur Ingénieur, Université Paul Sabatier, Toulouse (1980).

[25] Chartagnac, P., Réalisation et étalonnage de capteurs piézorésistifs de basse impédance. Description des chaînes d'alimentation et de mesures associées. Rapport technique CEG, avril 1983 (non publié).

[26] Bernstein, D. et KeOugh, D. D., Piezoresistivity of manganin, J. Appl. Phys. 37 no 5 (1964).
[27] Fuller, P. J. A. et Price, J. H., Dynamic pressure measurements to 300 kilobar with a resistance transducer, Brit. J. Appl. Phys. 15 (1964).

[28] Gagnoux, J., Shock wave compression of a borosilicate glass to $170 \mathrm{Kar}, 1981$ Topical Conference on Shock waves in condensed matter, SRI International, MENLO Park, CA, USA (1981).

[29] Chartagnac, P., Determination of mean and deviatoric stresses in shock loaded solids, J. Appl. Phys. 53 (2) (1982).

[30] Chartagnac, P., Loupias, C., Jimenez, B., Mesure continue de la vitesse particulaire par «jauges électromagnétiques » au sein d'un solide en régime de choc plan, Rapport technique CEG (Septembre 1982) (non publié).

[31] Barker, L. M., Hollenbach, R. E., J. Appl. Phys. 43 (1972) 4669.

[32] Chartagnac, P., Mesure de la vitesse de surface libre d'une cible soumise à un choc. Introduction à la méthode interférométrique DOPPLER-LASER du type « VISAR ». Rapport technique CEG (Novembre 1980) (non publié).

[33] Wilkins, L., GuINAN, M., Impact of cylinders on a rigid boundary, J. Appl. Phys. 44 (1973) 1200.

[34] Assistance Industrielle Dauphinoise, Z.I. Chemin des clos 38240 Meylan. 